\title{
Nilpotency and Limit Sets of Cellular Automata
}

\author{
Pierre Guillon ${ }^{1}$ and Gaétan Richard ${ }^{2}$ \\ 1 Université Paris-Est \\ Laboratoire d'Informatique Gaspard Monge, UMR CNRS 8049 \\ 5 bd Descartes, 77454 Marne la Vallée Cedex 2, France \\ pierre.guillon@univ-mlv.fr \\ 2 Laboratoire d'Informatique Fondamentale de Marseille \\ Aix-Marseille Université, CNRS \\ 39 rue Joliot-Curie, 13013 Marseille, France \\ gaetan.richard@lif.univ-mrs.fr
}

\begin{abstract}
A one-dimensional cellular automaton is a dynamical system which consisting in a juxtaposition of cells whose state changes over discrete time according to that of their neighbors. One of its simplest behaviors is nilpotency: all configurations of cells are mapped after a finite time into a given "null" configuration. Our main result is that nilpotency is equivalent to the condition that all configurations converge towards the null configuration for the Cantor topology, or, equivalently, that all cells of all configurations asymptotically reach a given state.
\end{abstract}

Keywords: cellular automata, nilpotency, dynamical systems, attractors

\section{Introduction}

Discrete dynamical systems aim at representing evolutions of objects in astronomy, chemistry, cellular biology, zoology, computing networks... Evolutions of these objects can often be described by iterations of a continuous function. The sequence of values obtained is called orbit.

A long-standing issue in dynamical systems is the distinction between limit behavior and finitely-reached behavior: in which case does convergence of orbits imply reaching the limit in finite time? Of course, there are obvious examples in which all orbits converge towards the same limit without ever reaching it, such as the division by 2 on segment $[0,1]$.

Here, we limit our study to some particular systems: cellular automata (CA). A CA consists in an infinite number of identical cells arranged on a regular lattice. All cells evolve synchronously according to their own state and those of their neighbors. It is thus a dynamical system on the set of configurations (which map each cell of the lattice to some state).

Endowing the set of configurations with the product topology allows the following restatement of the above-mentioned issue: in which case can local behavior (evolution of a particular cell) be uniformed into global behavior (evolution

* This work has been supported by the ANR Blanc "Projet Sycomore". 
of the whole configuration)? This question can also be seen as a comparison between the limit set (configurations that can be reached arbitrarily late) and the ultimate set (adjacent values of orbits).

In this article, we study in detail the nilpotency of $\mathrm{CA}$, in which all configurations eventually reach a given uniform configuration. Even though it represents the simplest behavior a dynamical system can have, its relevance grew in the CA field when Jarkko Kari made it the prototype of the undecidable problem on dynamics of CA [1], which has been widely used in reductions since then. This paper is dedicated to the equivalence between nilpotency and convergence toward a given configuration, or nilpotency of the trace subshift, which is a dynamical system linked to the CA, studied in $[2,3]$, and which represents the observation of the evolution of a cell by a fixed observer. In other words, in that case, the local behavior can be uniformed.

The restriction to the case of CA with a spreading state is simpler and was already useful in some reductions (see for instance [3]). Here, we extend the result to all one-dimensional CA.

Section 2 is devoted to definitions and preliminary results. In Sect. 3, we recall some results about the nilpotency, and prove that uniform configurations are isolated in the limit set only if the CA is nilpotent. Sect. 4 is devoted to proving our new characterization of nilpotency and Sect. 5 gives some new tracks of generalization.

\section{Preliminaries}

Let $\mathbb{N}^{*}=\mathbb{N} \backslash\{0\}$. For $i, j \in \mathbb{N}$ with $i \leq j,[i, j]$ (resp. $] i, j[$ ) denotes the set of integers between $i$ and $j$ inclusive (resp. exclusive). For any function $F$ from $A^{\mathbb{Z}}$ into itself, $F^{n}$ denotes the $n$-fold composition of $F$ with itself.

Words. Let $A$ be a finite alphabet with at least two letters. A word is a finite sequence of letters $w=w_{0} \ldots w_{|w|-1} \in A^{*}$, where $|w|$ is the length of $w$. A factor of a word $w=w_{0} \ldots w_{|w|-1} \in A^{*}$ is a word $w_{[i, j]}=w_{i} \ldots w_{j}$, for $0 \leq i \leq j<|w|$.

\subsection{Dynamical systems}

A (discrete) dynamical system (DS for short) is a couple $(X, F)$, where $X$ is a compact metric space, and $F$ is a continuous function. When no confusion is possible (especially when $X=A^{\mathbb{Z}}$ ), $X$ will be omitted. The orbit of initial point $x \in X$ is the sequence of points $F^{j}(x)$ when generation $j \in \mathbb{N}$ grows (where $F^{j}$ denotes the $j$-fold composition of $F$ with itself). We note $\mathcal{O}_{F}(x)=$ $\left\{F^{j}(x) \mid j \in \mathbb{N}\right\}$. We say that $Y \subset X$ is $F$-stable and that $(Y, F)$ is a subsystem of $(X, F)$ if $F(Y) \subset Y$.

Asymptotic behavior of orbits is represented by two particular sets:

- the $\left(\Omega\right.$-) limit set of a DS $(X, F)$ is the set $\Omega_{F}=\bigcap_{j \in \mathbb{N}} F^{j}(X)$ of all configurations that can appear arbitrarily late in orbits. 
- The ultimate set is the set $\omega_{F}=\bigcup_{x \in X} \bigcap_{J \in \mathbb{N}} \overline{\mathcal{O}_{F}\left(F^{J}(x)\right)}$ of all adhering values of orbits.

One can notice that both are $F$-stable sets, and that, by definition, $\omega_{F} \subset \Omega_{F}$, whereas the converse is generally false. Moreover, compactness implies the nonemptiness of those two sets, and that the limit and ultimate sets of any DS F are equal to those of any of its iterated $F^{k}$ for $k \in \mathbb{N}^{*}$.

\section{$2.2 \quad$ Space $A^{\mathbb{Z}}$}

Let $A$ be a finite alphabet. The set $A^{\mathbb{Z}}$ (resp. $A^{\mathbb{N}}$ ) is the set of bi-infinite (resp. infinite) sequences over alphabet $A$. An element $x \in A^{\mathbb{Z}}$ is called configuration (see Fig. 1a). A factor of $x$ is a word $x_{[i, j]}=x_{i} \ldots x_{j}$, for $i \leq j$. This notion of factor can be extended to infinite intervals (such as $]-\infty, j]$ or $[i,+\infty[$ ). The notation $x_{<i>}$ stands for the central factor $x_{[-i, i]}$.

Topology. $A^{\mathbb{Z}}$ is endowed with the product (or Cantor) topology, corresponding to the distance

$$
\begin{aligned}
A^{\mathbb{Z}} \times A^{\mathbb{Z}} & \rightarrow \mathbb{R}_{+} \\
(x, y) & \mapsto 2^{-\min _{x_{i} \neq y_{i}}|i|},
\end{aligned}
$$

which makes it compact, perfect, totally disconnected. A similar distance can be defined on $A^{\mathbb{N}}$. An example of two configuration at distance $2^{-2}$ is depicted in Fig. 1b.

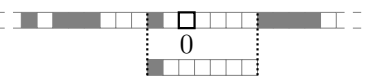

(a) Configuration $c \in A^{\mathbb{Z}}$ and $c_{[-2,5]}$

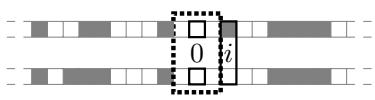

(b) Two configurations at distance $2^{-i}$

Fig. 1. Elements of $A^{\mathbb{Z}}$

Cylinders. For $j, k \in \mathbb{N}$ and a finite set $W$ of words of length $j$, we note $[W]_{k}$ the set $\left\{x \in A^{\mathbb{Z}} \mid x_{[k, k+j[} \in W\right\}$. Such a set is called a cylinder. Cylinders form a countable base of clopen sets.

We note $[W]_{k}{ }^{C}$ the complement of the cylinder $[W]_{k},[W]$ the center cylinder $[W]_{\left\lfloor\frac{j}{2}\right\rfloor}$, if $K \subset A^{\mathbb{N}},[W]_{k} K=\left\{x \in A^{\mathbb{Z}} \mid x_{[k, k+j[} \in W\right.$ and $\left.x_{[k+j, \infty[} \in K\right\}$, and,

if $K \subset A^{-\mathbb{N}}, K[W]_{k}=\left\{x \in A^{\mathbb{Z}} \mid x_{[k, k+j[} \in W\right.$ and $\left.x_{]-\infty, k[} \in K\right\}$. Similarly to what is done for languages, we will assimilate a singleton with its unique element; for instance, $z[u]_{i} z^{\prime}$ will denote the configuration $x \in A^{\mathbb{Z}}$ such that $x_{-\infty, i[}=z$, $x_{[i, i+|u|[}=u$ and $x_{i+|u|, \infty[}=z^{\prime}$. Finally, note that notations such as $z[] z^{\prime}$ will be used for the configuration $x \in A^{\mathbb{Z}}$ such that $x_{-\infty, 0[}=z$ and $x_{[0, \infty[}=z^{\prime}$. 
Finite configurations. If $q \in A$, then $q^{\omega}$ is the infinite word of $A^{\mathbb{N}}$ consisting in periodic repetitions of $q,{ }^{\omega} q^{\omega}$ is the (spatially) periodic configuration of $A^{\mathbb{Z}}$ consisting in repetitions of $q,{ }^{\omega} q^{\omega}$ is the $q$-uniform configuration, and any configuration ${ }^{\omega} q[u] q^{\omega}$, where $u \in A^{*}$, is a $q$-finite configuration. A q-semi-finite configuration is a configuration $x={ }^{\omega} q z$ for some $z \in A^{\mathbb{N}} \backslash\left\{q^{\omega}\right\}$. Note that the set of $q$-finite configurations is dense, since any finite word $u$ can be extended as ${ }^{\omega} q u q^{\omega} \in[u]$.

\subsection{Cellular automata}

A (one-dimensional two-sided) cellular automaton (CA for short) is a discrete dynamical system consisting in cells distributed over the regular lattice $\mathbb{Z}$. Each cell $i \in \mathbb{Z}$ of the configuration $x \in A^{\mathbb{Z}}$ has a state $x_{i}$ in the finite alphabet $A$. That state evolves according to the state of their neighbors: $\left.F(x)_{i}=f\left(x_{[i-r, i+r[}\right)\right)$, where $f: A^{2 r+1} \rightarrow A$ is the local rule and $r \in \mathbb{N}$ the radius of the CA. By abuse of notation, we assimilate the CA to its global function $F: A^{\mathbb{Z}} \rightarrow A^{\mathbb{Z}}$, which is a DS on the configurations space. Usually, an orbit is graphically represented by a two-dimensional space-time diagram, such as in Figure 2.

Example 1. A simple example of $\mathrm{CA}$ is the min $\mathrm{CA}$, defined on alphabet $A=$ $\{0,1\}$ by radius 1 and local rule

$$
\begin{aligned}
A^{3} & \rightarrow A \\
f:\left(x_{-1}, x_{0}, x_{1}\right) & \mapsto \mid \begin{array}{ll}
1 & \text { if } x_{-1}=x_{0}=x_{1}=1 \\
0 & \text { otherwise }
\end{array} .
\end{aligned}
$$

The typical evolution makes all finite bunches of 1s disappear progressively; only the infinite configuration ${ }^{\omega} 1^{\omega}$ will not lose any 1 and nearly all configurations tend towards ${ }^{\omega} 0^{\omega}$. The ultimate set is $\omega_{F}=\left\{{ }^{\omega} 0^{\omega},{ }^{\omega} 1^{\omega}\right\}$ and the limit set $\Omega_{F}$ is the set of configurations that do not contain patterns of the form $10^{k} 1$ for any $k \in \mathbb{N}^{*}$.

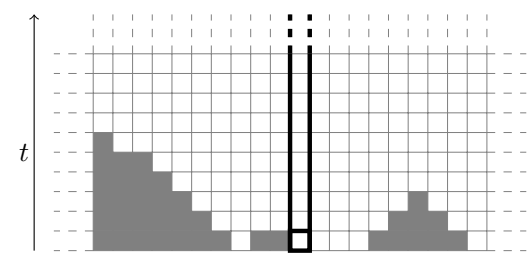

Fig. 2. A space-time diagram of the min $\mathrm{CA}$ with highlighted $r$-blocking word.

Quiescent states. A state $0 \in A$ is said to be quiescent for the CA $F$ with local rule $f$ if $f(0, \ldots, 0)=0$. Equivalently, $F\left({ }^{\omega} 0^{\omega}\right)={ }^{\omega} 0^{\omega}$, which is called a quiescent 
uniform configuration. For instance, both 0 and 1 are quiescent states for the $\min \mathrm{CA}$.

We can see that the set of uniform configurations form a finite, hence ultimately periodic, subsystem of the cellular automaton; hence $\forall a \in A, \exists j<$ $|A|, F^{|A|}\left({ }^{\omega} a^{\omega}\right)=F^{|A|-j-1}\left({ }^{\omega} a^{\omega}\right)$, which gives the following remark:

Remark 1. For any CA $F, F^{j}$ admits a quiescent state for some $j \in[1,|A|]$.

It can also easily be seen that the ultimate set of any CA contains all quiescent configurations. Since $\omega_{F^{j}}=\omega_{F}$, the previous remark gives, in particular, that for any CA $F$, there is at least one uniform configuration in $\omega_{F}$ (and therefore $\left.\Omega_{F}\right)$.

Blocking words. A word $w \in A^{*}$ is $(i, k)$-blocking (or simply $k$-blocking) for $\mathrm{CA} F: A^{\mathbb{Z}} \rightarrow A^{\mathbb{Z}}$ if $\forall x, y \in[w]_{-i}, \forall j \in \mathbb{N}, F^{j}(x)_{[0, k[}=F^{j}(y)_{[0, k[}$. If $r$ is the radius of $F$, note that if a word $w$ is $(i, r)$-blocking, and $x \in[w]_{-i}$, then for all configurations $y \in A^{\mathbb{Z}}$ such that $y_{[-i, \infty[}=x_{[-i, \infty[}$ (resp. $y_{-\infty,|w|-i[}=$ $\left.x_{-\infty,|w|-i[}\right)$, and for all generation $j \in \mathbb{N}$, we have $F^{j}(y)_{[0, \infty[}=F^{j}(x)_{[0, \infty[}$ (resp. $\left.F^{j}(y)_{-\infty, r[}=F^{j}(x)_{-\infty, r}\right)$, i.e. information cannot pass through an $r$ blocking word. For instance, in the min CA, the word 0 is $(0,1)$-blocking, since $\forall x \in[0], \forall j \in \mathbb{N}, F^{j}(x)_{0}=0$ (see Fig.2), hence space-time diagrams containing one 0 can be separated into two independent evolutions.

\subsection{Symbolic dynamics}

The shift map $\sigma: A^{\mathbb{Z}} \rightarrow A^{\mathbb{Z}}$ is a particular CA defined by $\sigma(x)_{i}=x_{i+1}$ for every $x \in A^{\mathbb{Z}}$ and $i \in \mathbb{Z}$ which shifts configurations to the left. Hedlund [4] has shown that cellular automata are exactly the DS on $A^{\mathbb{Z}}$ which commute with the shift.

This characterization leads us to study shift-invariant subsets of $A^{\mathbb{Z}}$, among which, for instance, the image or limit sets of any CA. With the help of topology, we shall give some useful properties on those sets.

Subshifts. The one-sided shift (or simply shift when no confusion is possible), also noted $\sigma$ by abuse of notation, is the self-map of $A^{\mathbb{N}}$ such that $\sigma(z)_{i}=z_{i+1}$, for every $z \in A^{\mathbb{N}}$ and $i \in \mathbb{N}$. A one-sided subshift $\Sigma \subset A^{\mathbb{N}}$ is a $\sigma$-stable closed set of infinite words. The following statements concern a two-sided version of subshifts.

Proposition 1. The only closed shift-stable subset of $A^{\mathbb{Z}}$ of nonempty interior is $A^{\mathbb{Z}}$.

Proof. Let $\Sigma$ be such a set. Having nonempty interior, it must contain some cylinder $[u]_{i}$ with $u \in A^{*}$ and $i \in \mathbb{Z}$. Being closed and shift-stable, it must also contain $\overline{\bigcup_{j \leq i} \sigma^{j}\left([u]_{i}\right)}=A^{\mathbb{Z}}$.

Corollary 1. If $A^{\mathbb{Z}}=\bigcup_{j \in \mathbb{N}} \Sigma_{j}$ where the $\Sigma_{j}$ are closed and shift-stable, then $A^{\mathbb{Z}}$ is equal to some $\Sigma_{j_{0}}$. 
Proof. $A^{\mathbb{Z}}$ being complete, and of nonempty interior, the Baire Theorem states for some $j_{0} \in \mathbb{N}, \Sigma_{j_{0}}$ has nonempty interior too. By Proposition $1, \Sigma_{j_{0}}=A^{\mathbb{Z}}$.

\subsection{Trace}

Rather than observing the whole configuration, trace consists in observing a specific portion of configuration during evolution of a cellular automaton. This notion establishes a link between the theory of cellular automata and symbolic dynamics.

Definition 1 (Trace). Given a $C A F$, the trace of $F$ with initial condition $x \in A^{\mathbb{Z}}$ is $T_{F}(x)=\left(F^{j}(x)_{0}\right)_{j \in \mathbb{N}}$. In other words, it is the central column of the space-time diagram of initial configuration $x$ (see Figure 3). The trace subshift of $F$ is the image set $\tau_{F}=T_{F}\left(A^{\mathbb{Z}}\right)$. Similarly, we define $T_{F}^{k}(x)=\left(F^{j}(x)_{[0, k[}\right)_{j \in \mathbb{N}}$, the sequence of the words at positions in $[0, k[$ in the space-time diagram of initial configuration $x$, and $\tau_{F}^{k}=T_{F}^{k}\left(A^{\mathbb{Z}}\right)$.

For instance, if $A=\{0,1\}$, then the trace subshift is $(0+1)^{\omega}$ for the shift, $0^{\omega}+1^{\omega}$ for the identity and $(0+1) 0^{\omega}$ for the CA that maps every cell to 0 (see [2] for more examples).

Note that $T_{F} F=\sigma T_{F}$ and, as $T_{F}$ is continuous, we say it is a factorization between the CA and the subshift $\left(\tau_{F}, \sigma\right)$ which means that their two dynamics are very closely related. For more about factorizations, see [5]. In the following, $\tau_{F}$ and $\tau_{F}^{k}$ may stand for the dynamical systems $\left(\tau_{F}, \sigma\right)$ and $\left(\tau_{F}^{k}, \sigma\right)$.

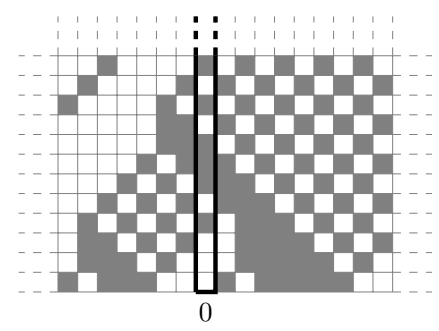

Fig. 3. Trace seen on the space-time diagram

In this paper, we try to obtain links between the set of possible traces and the global behavior of the cellular automaton. That is try to "globalize" a local observation of the behavior.

\section{$3 \quad$ Nilpotent cellular automata}

In this section, we recall the definition and characterizations of cellular automata nilpotency, and its known link with the limit set. We present a short proof empha- 
sizing on the fact that a CA is nilpotent as soon as some uniform configuration is isolated in its limit set.

Definition 2. A $D S(X, F)$ is weakly nilpotent if there is a particular "null" configuration $z \in X$ such that for every point $x \in X$, there is a generation $J \in \mathbb{N}$ such that $\forall j>J, F^{j}(x)=z$. The system is said nilpotent if the generation $J$ does not depend on the point, i.e. $\exists J \in \mathbb{N}, \forall x \in X, \forall j>J, F^{j}(x)=z$.

Let us now consider the specific case of cellular automata. If a CA $F$ is weakly nilpotent, then, from the definition, the null configuration must be $F$ invariant. Moreover, for any $z \in A^{\mathbb{Z}}$, there is some $j$ for which, on the one hand $F^{j}(\sigma(z))=z$, and on the other hand, $F^{j}(\sigma(z))=\sigma\left(F^{j}(z)\right)=\sigma(z)$. Hence $z=\sigma(z)$ is a uniform quiescent configuration, i.e. $z={ }^{\omega} 0^{\omega}$ for some quiescent state 0 . One can also make the following easy remarks:

Remark 2.

- Suppose $F$ is a $C A$ and $j \in \mathbb{N}$ a generation such that for all configurations $x \in$ $A^{\mathbb{Z}}, F^{j}(x)_{0}=0$. Then shift-invariance of $A^{\mathbb{Z}}$ gives $A^{\mathbb{Z}}=\bigcap_{k \in \mathbb{Z}} \sigma^{k} F^{-j}([0])$, and by commuting $F$ and the shift, we obtain $A^{\mathbb{Z}}=F^{-j}\left({ }^{\omega} 0^{\omega}\right)$. Hence $F$ is nilpotent.

- On the other hand, if $F$ is not nilpotent, then $F^{-j}\left(\left[0^{C}\right]\right)$ is a nonempty open set; in particular, it contains some $q$-finite configurations, for any state $q \in A$.

- Note that if $F$ is a non-nilpotent CA, from Remark 1 , there is some $J \in \mathbb{N}$ such that some $0 \in A$ is quiescent for $F^{J}$. We can see that the set of 0 -finite configurations is $F^{J}$-stable. Hence, from the previous point, for all $j \in \mathbb{N}$, $F^{j}\left(A^{\mathbb{Z}}\right)$ contains some non-uniform 0-finite configuration $z={ }^{\omega} 0[u] 0^{\omega}$ with $u \in A^{*}$ and $z_{0} \neq 0$.

Here is a precise characterization of the limit set of nilpotent CA.

Theorem 1 (Čulik, Pachl, Yu [6]). A CA whose limit set is a singleton is nilpotent.

Proof. If $F$ is a CA such that $\Omega_{F} \cap\left[0^{C}\right]=\emptyset$, the intersection $\bigcap_{j \in \mathbb{N}}\left(F^{j}\left(A^{\mathbb{Z}}\right) \cap\left[0^{C}\right]\right)$ of closed sets is empty. By compactness, $F^{j}\left(A^{\mathbb{Z}}\right) \cap\left[0^{C}\right]$ is empty for some $j \in \mathbb{N}$. $F^{j}\left(A^{\mathbb{Z}}\right)$ being a subshift, we conclude that $F^{j}\left(A^{\mathbb{Z}}\right) \cap\left(\bigcup_{i \in \mathbb{Z}}\left[0^{C}\right]_{i}\right)=F^{j}\left(A^{\mathbb{Z}}\right) \backslash^{\omega} 0^{\omega}$ is empty.

With this characterization and the fact that $\Omega_{F}=\Omega_{F^{j}}$, we have the following corollary.

Corollary 2. A CA F is nilpotent if and only if so is some (so are all) of its iterated $C A F^{n}$, for $n \in \mathbb{N}$.

The next allows to prove the well-known equivalence between nilpotency and weak nilpotency. 
Proposition 2. If for every configuration $x \in A^{\mathbb{Z}}$, there is a generation $j \in \mathbb{N}$ and a cell $i \in \mathbb{Z}$ such that $F^{j}(x)_{[i, \infty[}=0^{\omega}\left(\right.$ resp. $\left.F^{j}(x)_{]-\infty, i]}={ }^{\omega} 0\right)$, then $F$ is nilpotent.

Proof. Assume $A^{\mathbb{Z}}=\bigcup_{j \in \mathbb{N} i \in \mathbb{Z}} \sigma^{i}\left(F^{-j}\left({ }^{\omega} A\left[0^{\omega}\right)\right)\right.$ (the other case is symmetric). From Corollary $1, A^{\mathbb{Z}}=\sigma^{i}\left(F^{-j}\left({ }^{\omega} A\left[0^{\omega}\right)\right)\right.$ for some generation $j \in \mathbb{N}$ and some $i \in \mathbb{Z}$. In particular, $\sigma^{i}\left(A^{\mathbb{Z}}\right)=F^{-j}([0])$, and Remark 2 allows to conclude the claim.

Corollary 3. A weakly nilpotent $C A$ is nilpotent.

There is indeed some "gap" between the possible evolutions of nilpotent and non-nilpotent CA. This is also stressed by the following result on limit set: it is sufficient that the it admits an isolated uniform configuration for the CA to be nilpotent.

Proposition 3. If $F$ is a non-nilpotent $C A$, then $\Omega_{F}$ contains some semi-finite configuration ${ }^{\omega} 0[] z$, for some $0 \in A$ and some $z \in A^{\mathbb{N}} \backslash\left\{0^{\omega}\right\}$.

Proof. Let $F$ be a non-nilpotent CA. Should we iterate it, we can suppose it has a quiescent state $0 \in A$, thanks to Remark 1 and Corollary 2. By Remark 2, for every generation $j \in \mathbb{N}, F^{j}\left(A^{\mathbb{Z}}\right) \cap{ }^{\omega} 0\left[0^{C}\right] \neq \emptyset$, and compactness gives that $\Omega_{F} \cap{ }^{\omega} 0\left[0^{C}\right] \neq \emptyset$.

Theorem 2. The limit set of any non-nilpotent CA does not have any isolated uniform configurations.

Proof. Let $F$ a non-nilpotent CA, $0 \in A, k \in \mathbb{N}$ and $x \in{ }^{\omega} 0[] \cap \Omega_{F} \backslash\left\{{ }^{\omega} 0^{\omega}\right\}$ as in Proposition 3. Then, for any $k \in \mathbb{N}, \sigma^{-k}(x)$ is a configuration of $\Omega_{F}$ distinct from ${ }^{\omega} 0^{\omega}$, and which is in the cylinder $\left[0^{2 k+1}\right]$ around ${ }^{\omega} 0^{\omega}$.

Remark that, on the contrary, $\omega_{F}$ can have isolated uniform configurations even in the non-nilpotent case. For instance, the ultimate set of the min CA is $\left\{{ }^{\omega} 0^{\omega},{ }^{\omega} 1^{\omega}\right\}$.

The previous result allows state that limit sets are either a singleton or infinite.

Corollary 4 (Čulik, Pachl, Yu [6]). The limit set of any non-nilpotent CA is infinite.

Note that the limit set can be numerable, as for the min CA, or innumerable, as for the shift CA.

To sum up, the limit set of a CA has a very precise structure, and, as will be seen more deeply in the following section, constraining a little can make it collapse to a singleton, i.e. make the $\mathrm{CA}$ nilpotent. 


\section{Nilpotent traces}

We have seen in the previous section that there is a "gap" between nilpotent and non-nilpotent CA in terms of the limit set. The aim of this section is to prove that it is also the case for the ultimate set, or, equivalently, for the trace subshift: a constraint such as the ultimate set being a singleton is restrictive enough to imply nilpotency. We first give a directly proved characterization of that notion for trace subshifts.

Remark 3. Similarly to the case of CA, we can notice that a subshift $\Sigma \in A^{\mathbb{N}}$ is weakly nilpotent if and only if there is some state $0 \in A$ such that for every infinite word $w \in \Sigma$, there is some $j \in \mathbb{N}$ such that $w_{[j, \infty[}=0^{\omega}$.

The previous remark will justify the concept of 0 -nilpotency, where 0 is a quiescent state. We also say that some configuration $x$ is 0 -mortal for the CA $F$ if there exists $j \in \mathbb{N}$ such that $F^{j}(x)=0^{\omega}$.

Proposition 4. Let $F$ a $C A$. The following statements are equivalent:

1. $\tau_{F}$ is weakly 0-nilpotent.

2. $\tau_{F}^{k}$ is weakly $0^{k}$-nilpotent, for any integer $k \in \mathbb{N}^{*}$.

3. For every configuration $x \in A^{\mathbb{Z}}, F^{n}(x)$ tends, as $n \rightarrow \infty$, to the same "null" configuration ${ }^{\omega} 0^{\omega}$.

4. $\omega_{F}=\left\{{ }^{\omega} 0^{\omega}\right\}$.

Let us prove now that, in the same way as weakly nilpotent CA are nilpotent, weakly nilpotent traces are nilpotent, i.e. the corresponding CA is nilpotent (at least in dimension 1).

The proof is organized the following way: we first prove that a $\mathrm{CA}$ with weakly nilpotent trace has an $r$-blocking word, and then that there exists a bound on the converging time for mortal finite configurations. At last, we exhibit a contradiction with a non-nilpotency hypothesis.

Lemma 1. Let $F$ a $C A$ whose trace is weakly 0 -nilpotent; then for every $k \in \mathbb{N}$, there is a generation $J \in \mathbb{N}$ such that for every configuration $x \in A^{\mathbb{Z}}, \exists j \leq$ $J, F^{j}(x) \in\left[0^{k}\right]$.

Proof. Let $F$ a CA such that $\tau_{F}$ is weakly nilpotent and $k \in \mathbb{N}$. By Point 2 of Proposition 4 and Remark 3, we have $A^{\mathbb{Z}}=\bigcup_{j \in \mathbb{N}} F^{-j}\left(\left[0^{k}\right]\right)$. By compactness, we can extract a finite covering $A^{\mathbb{Z}}=\bigcup_{j \leq J} F^{-j}\left(\left[0^{k}\right]\right)$ for some $J \in \mathbb{N}$.

Lemma 2. If $F$ is a CA with a nilpotent trace, then it admits an r-blocking word.

Proof. Let $F$ such a CA. By Point 2 of Proposition $4, A^{\mathbb{Z}}=\bigcup_{J \in \mathbb{N}} \bigcap_{j>J} F^{-j}\left(\left[0^{r}\right]\right)$ has nonempty interior, for some $0 \in A$; hence the Baire Theorem states that there is some $J \in \mathbb{N}$ for which $\bigcap_{j>J} F^{-j}\left(\left[0^{r}\right]\right)$ has nonempty interior; it contains a cylinder $[u]_{-i}$ for some $u \in A^{*}$ and $i \in \mathbb{N}$. We can assume without loss of 
generality that $i \geq J r$ and $|u| \geq(J+1) r+i$, which gives $\forall x, y \in[u]_{-i}, \forall j \leq$ $J, F^{j}(x)_{[0, r[}=F^{j}(y)_{[0, r[}$, and we already have by construction that $\forall x, y \in$ $[u]_{-i}, \forall j>J, F^{j}(x)_{[0, r[}=0^{r}=F^{j}(y)_{[0, r[}$. Hence, $u$ is an $(i, r)$-blocking word.

Lemma 3. Let $F$ a CA whose trace is weakly 0-nilpotent; then there is a generation $j$ for which any 0 -finite 0 -mortal configuration $x$ satisfies $F^{j}(x)={ }^{\omega} 0^{\omega}$.

Proof. Consider $F$ a CA such that $\tau_{F}$ is weakly nilpotent, and for all $j \in \mathbb{N}$, there is a finite mortal configuration $y$ such that $F^{j}(y) \neq{ }^{\omega} 0^{\omega}$. Should it be shifted, we can suppose $F^{j}(y)_{0} \neq 0$.

Claim. For every $k \in \mathbb{N}$, there is a 0 -finite 0 -mortal configuration $y \in\left[0^{2 k+1}\right]$ and a generation $j \geq k$ such that $F^{j}(y)_{0} \neq 0$.

Proof. Let $k \in \mathbb{N}$. From Lemma $1, \exists J \in \mathbb{N}, \forall y \in A^{\mathbb{Z}}, \exists j \leq J, F^{j}(y) \in\left[0^{2 k+1}\right]$. By hypothesis, there exists a finite mortal configuration $x$ for which $F^{J}(x)_{0} \neq 0$. Hence, $\exists j \leq J, F^{j}(x) \in\left[0^{2 k+1}\right]$. Just take the configuration $y=F^{j}(x)$; we have $F^{J-j}(y)_{0}=F^{J}(x)_{0} \neq 0$. Of course, $\forall j<k, F^{j}(y)_{0}=0$.

Claim. Let $x$ a 0 -finite 0 -mortal configuration and $k \in \mathbb{N}$. Then, there is a 0 -finite 0 -mortal configuration $y \in\left[x_{<r k>}\right]$ and

$$
\left\{j \in \mathbb{N} \mid F^{j}(y)_{0} \neq 0\right\} \supsetneq\left\{j \in \mathbb{N} \mid F^{j}(x)_{0} \neq 0\right\} .
$$

Proof. Let $x$ a finite configuration and $n \in \mathbb{N}$ such that $F^{n}(x)={ }^{\omega} 0^{\omega}$. Should we take a larger $k$, we can assume that $x \in{ }^{\omega} 0\left[A^{2 r(k-2 n)}\right] 0^{\omega}$. In particular, note that $k \geq 2 n$. By the previous claim, there exists a finite mortal configuration $x^{\prime} \in\left[0^{2 k+1}\right]$ and a generation $j \geq k$ such that $F^{j}(x)_{0} \neq 0$. Let $y=x_{-\infty,-r k[}^{\prime}\left[x_{<r k>}\right] x_{1 r k, \infty[}^{\prime}$. We can easily see by induction on $j \leq n$ that $F^{j}(y) \in F^{j}\left(x^{\prime}\right)_{-\infty,-r(k-2 n+j)[}\left[A^{2 r j} F^{j}(x)_{<r(k-j)>} A^{2 r j}\right] F^{j}\left(x^{\prime}\right)_{r(k-2 n+j), \infty[}$. In particular, on the one hand, $\left\{j \in \mathbb{N} \mid F^{j}(y)_{0} \neq 0\right\} \cap[0, n]=\left\{j \in \mathbb{N} \mid F^{j}(x)_{0} \neq 0\right\} \cap$ $[0, n]=\left\{j \in \mathbb{N} \mid F^{j}(x)_{0} \neq 0\right\}$. On the other hand, since $F^{n}(x)_{<r(k-n)>}=0^{2 r(k-n)}=$ $F^{n}\left(x^{\prime}\right)_{<r(k-n)>}$, we get $F^{n}(y)=F^{n}\left(x^{\prime}\right)$. By construction, there is a generation $j \geq k>n$ such that $F^{j}(y)_{0}=F^{j}\left(x^{\prime}\right)_{0} \neq 0$. To sum up, $\left\{j \in \mathbb{N} \mid F^{j}(y)_{0} \neq 0\right\} \supsetneq$ $\left\{j \in \mathbb{N} \mid F^{j}(x)_{0} \neq 0\right\}$.

We can now build by induction a sequence $\left(y^{k}\right)_{k \in \mathbb{N}}$ of finite mortal configurations, with $x^{0}={ }^{\omega} 0^{\omega}$, and such that for $k \in \mathbb{N}, x^{k+1} \in\left[x_{<r(k+1)>}^{k}\right]$ and $\left\{j \in \mathbb{N} \mid F^{j}\left(x^{k+1}\right)_{0} \neq 0\right\} \supsetneq\left\{j \in \mathbb{N} \mid F^{j}\left(x^{k}\right)_{0} \neq 0\right\}$. That Cauchy-Bolzano sequence of finite mortal configurations tends to some configuration $x \in A^{\mathbb{Z}}$ such that $\left\{j \in \mathbb{N} \mid F^{j}(x)_{0} \neq 0\right\}$ is infinite (by continuity of the trace), i.e. $\tau_{F}$ is not nilpotent.

Lemma 4. Let $F$ a one-dimensional non-nilpotent $C A$ admitting a weakly 0nilpotent trace and an $r$-blocking word. Then for every generation $j \in \mathbb{N}$, there exists some 0 -finite 0 -mortal configuration y such that $F^{j}(y) \neq{ }^{\omega} 0^{\omega}$. 
Proof. Let $F$ such a CA, $u$ a $(i, r)$-blocking word and $j \in \mathbb{N}$. By blockingness and Point 2 of Proposition 4, there is a generation $k \in \mathbb{N}$ such that $\forall n \geq k, \forall z \in$ $[u]_{-i}, F^{n}(z) \in\left[0^{r}\right]_{0}$. Should we take a strict superword for $u$, we can assume $|u|=2 i+1$. Remark 2 states that there is some finite configuration $x$ such that $F^{j+k}(x)_{0} \neq 0$. Consider the configuration $x^{\prime}={ }^{\omega} 0\left[u x_{<r(j+k)>} u\right] 0^{\omega}$, and the configuration $y={ }^{\omega} 0\left[F^{k}\left(x^{\prime}\right)_{<r(j+k)+i>}\right] 0^{\omega}$. By construction, $F^{j}(y)_{0}=F^{j+k}(x)_{0} \neq$ 0 . By induction on the generation $n \in \mathbb{N}, F^{n+k}\left(x^{\prime}\right) \in\left[0^{r} A^{2 r(j+k)+2 i+1} 0^{r}\right]$, and thus $F^{n}(y) \in{ }^{\omega} 0\left[A^{2 r(j+k)+2 i+1}\right] 0^{\omega}$. By hypothesis and Point 2 of Proposition 4, there is some generation $l \in \mathbb{N}$ such that $F^{l}(y) \in\left[0^{2 r(j+k)+2 i+1}\right]$, which gives $F^{l}(y)={ }^{\omega} 0^{\omega}$.

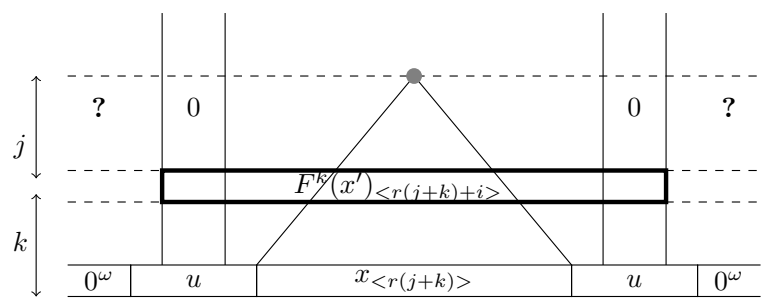

Fig. 4. Construction of mortal finite configurations with arbitrary slow convergence

Theorem 3. Any one-dimensional CA whose trace is weakly nilpotent is nilpotent.

Proof. Suppose there is a non-nilpotent CA $F$ such that $\tau_{F}$ is nilpotent. Then, by Lemma 2, we know that there exists an $r$-blocking word. Lemmas 4 and 3 give a contradiction.

Corollary 5. The subshift $\left(\varepsilon+0^{*} 1\right) 0^{\omega}$ is not the trace of any one-dimensional $C A$.

\section{Conclusion}

To sum up, for a one-dimensional CA $F$, the following statements are equivalent:

$F$ is nilpotent $\Leftrightarrow F$ is weakly nilpotent

$\Leftrightarrow \tau_{F}^{k}$ is nilpotent $\Leftrightarrow \tau_{F}^{k}$ is weakly nilpotent

$\Leftrightarrow \Omega_{F}$ is finite $\quad \Leftrightarrow \Omega_{F}$ has some isolated uniform configuration

$\Leftrightarrow \Omega_{F}$ is a singleton $\Leftrightarrow \omega_{F}$ is a singleton

We can remark that the nilpotency of a CA associated to a local rule does not depend on the anchor. Hence, all characterizations work up to a shift of the rule. 
The main open question is of course the generalization of these result to CA in upper dimensions (configurations in $A^{\mathbb{Z}^{d}}, d>1$ ). Juxtaposing blocking words (Lemma 4) is the crucial part which is difficult to transpose in dimension 2.

Another one is whether it is sufficient to take as a hypothesis that all orbits have ${ }^{\omega} 0^{\omega}$ as an adhering value, instead of a limit - i.e. all space-time diagrams walk through cylinders $\left[0^{k}\right]$ for any $k \in \mathbb{N}$ - to conclude the CA is nilpotent.

A natural question is whether the characterization of nilpotency in terms of trace can be generalized, for instance to preperiodicity (i.e. ultimate periodicity), in the same way as the finite-time characterization of nilpotency (Corollary 3; see for instance [5]). This is untrue; some CA $F$ may have all the infinite words of its trace preperiodic (i.e. $\forall z \in \tau_{F}, \exists p, q \in \mathbb{N}, \sigma^{p+q}(z)=\sigma^{q}(z)$ ), but with an unbounded preperiod, such as the min CA, whose trace subshift $1^{\omega}+1^{*} 0^{\omega}$ has only preperiodic words, but with an arbitrarily high preperiod.

Note that our result can be seen as a constraint on the structure of limit sets of non-nilpotent CA. In that idea, Proposition 3 allows to state that a CA is nilpotent as soon as its limit set contains only periodic configurations. Note that this is untrue for $\omega_{F}$ : for instance, the min CA has only spatially periodic configurations in its ultimate set. We can also mention another misleadingly simple issue, which concerns the presence of infinite configurations.

Conjecture 1. If $F$ is a $\mathrm{CA}$ and $0 \in A$ such that $\Omega_{F}$ contains only 0 -finite configurations, then $F$ is 0-nilpotent.

\section{Acknowledgement}

We gratefully thank Guillaume Theyssier, François Blanchard, Jarkko Kari for our very profitable discussions on that matter. Thanks also to our referees, who criticized constructively a rather unrefined version.

\section{References}

1. Kari, J.: The nilpotency problem of one-dimensional cellular automata. SIAM Journal on Computing 21(3) (1992) 571-586

2. Cervelle, J., Formenti, E., Guillon, P.: Sofic trace of a cellular automaton. In: CiE. Volume 4497 of Lecture Notes in Computer Science. (2007) 152-161

3. Cervelle, J., Guillon, P.: Towards a Rice theorem on traces of cellular automata. In Kučera, L., Kučera, A., eds.: MFCS. Volume 4708 of Lecture Notes in Computer Science. (2007) 310-319

4. Hedlund, G.A.: Endomorphisms and automorphisms of the shift dynamical system. Mathematical Systems Theory 3 (1969) 320-375

5. Kůrka, P.: Topological and symbolic dynamics. Société Mathématique de France (2003)

6. Culik II, K., Pachl, J., Yu, S.: On the limit sets of cellular automata. SIAM Journal on Computing 18(4) (1989) 831-842 